\title{
Le vaudeville des chants patriotiques dans La fille soldat de Desfontaines
}

Tomoko Nakayama, Université des Études étrangères de Kyoto

Dans son étude sur le répertoire des spectacles à Paris pendant la Révolution, André Tissier écrit : «Les œuvres les plus jouées ne sont pas celles des auteurs "politiques”. Les auteurs les plus joués sont des auteurs de la seconde partie du dix-huitième siècle, et, parmi ceux de la fin du siècle, des "amuseurs" sans prétention politique et philosophique » (524). Desfontaines, auteur de la pièce La fille soldat, est justement l'un de ces «amuseurs » qui ravissaient le public parisien. Entre septembre 1792 et octobre 1795, (c'est-à-dire dans la période qui va de la proclamation de la République à la fin de la Convention nationale), Desfontaines fut le deuxième auteur le plus joué, avec mille soixante-dix représentations, après Radet, mille trois cent trente-deux représentations (Tissier 523). François-Georges Fouques Deshayes dit Desfontaines (1733-1825) était un auteur prolifique, avec à son actif environ soixante-dix pièces, seul ou en collaboration, la plupart mêlées de vaudevilles. L'objet de notre article n'est pas de réhabiliter cet auteur aujourd'hui totalement oublié, ni de remettre à l'honneur la qualité littéraire de sa plume, mais, à partir d'une de ses pièces, d'examiner comment le contexte social de l'époque révolutionnaire a pu influencer un théâtre destiné au grand public.

\section{Le Théâtre du Vaudeville sous la Révolution}

La fille soldat fut créée au Théâtre du Vaudeville dont Desfontaines était l'un des auteurs fidèles. L'ouverture du Vaudeville était attendue du public parisien. Déjà en avril 1791, neuf mois avant l'inauguration du théâtre, « MM. Piis et Barré ont conçu l'idée d'ouvrir un asile au genre de vaudeville, que la musique italienne, le drame et les pièces dites à sentiment ont exclu depuis quelques années de tous nos grands théâtres, et qui cependant est analogue au génie de la nation » annonce un article de presse (Gazette nationale, 100). C'est le 12 janvier 1792 qu'on inaugura ce nouveau temple de vaudeville dans l'ancienne salle du Panthéon de la rue de Chartres.

Un autre article du Journal de Paris, quatre jours après l'ouverture du théâtre, explique ainsi la raison de l'attente du public : « on doit désirer, surtout, de voir la gaieté renaître \& adoucir les esprits, qu'une grande révolution a mis depuis deux ans dans une fermentation qui pourrait devenir dangereuse. Tout le monde sait par cœur les jolis couplets que MM. Piis \& Barré ont fait 
entendre il y a quelques années sur le Théâtre de la Comédie Italienne ; leur nom fut un augure heureux pour l'entreprise que nous annonçons »(66). Le Théâtre du Vaudeville eut un succès marquant et durable, au point qu'il devint « l'un des rendez-vous les plus populaires de Paris » et qu'il dura jusqu'en 1838, ayant «le rare mérite de ne pas changer de nom durant toute la Révolution » (Carlson 142). Selon l'étude de Tissier, «Du 21 septembre 1792 au 26 octobre 1795, période ici répertoriée, il y fut créé 85 pièces, et en fut repris 32. Et certaines pièces, dans ce laps de temps, eurent plus de cinquante représentations [...]» (181). Plus d'un tiers de nouvelles pièces ont été reprises. Le nombre de représentations des pièces nous montre que les auteurs connaissaient le goût du public et que le théâtre avait une assistance fidèle.

Le Théâtre du Vaudeville, ouvert à l'attente du public, a eu une histoire tumultueuse avec l'autorité. La chaste Suzanne, par exemple, écrite en collaboration de Barré, Desfontaines et Radet, et créée le 5 janvier 1793, a eu un grand succès auprès du public mais s'est vu interdire par la Convention qui y voyait des allusions satiriques à l'actualité politique. Cette décision entraîna l'arrestation des auteurs de La chaste Suzanne. Sous la Terreur, les auteurs dramatiques et les interprètes eurent du mal à se livrer à la création de leur gré et « la production dramatique [...] tendit de plus en plus à la pure propagande »(Carlson 200). Après six semaines d'emprisonnement dû à La chaste Suzanne, Barré, directeur du Vaudeville, fut libéré mi-octobre 1793. À la suite de la création d'une pièce patriotique, $L$ 'heureuse décade, il s'évertuera à faire libérer Desfontaines et Radet, en montant le 4 novembre la pièce Au retour, «drame ultra-révolutionnaire » (218) que Desfontaines et Radet avaient écrit en prison. Les artistes de spectacle étaient constamment obligés de montrer leur dévouement à la cause nationale par leurs propres œuvres. Le fait que la création des pièces de Desfontaines catégorisées comme «fait historique et patriotique » (Au retour et Encore un curé en novembre 1793, Les Chouans de Vitré en juin 1794) se concentre à cette période de la Terreur, témoigne le souci de l'auteur et de sa compagnie de complaire au pouvoir. À cette liste des pièces patriotiques, s'ajoutera La Fête de l'Égalité, créée le 25 février 1794, comédie ayant pour décor les bustes des martyrs de la liberté (Jean-Paul Marat et Louis-Michel Lepelletier), dans laquelle Desfontaines et Radet font l'éloge de Rousseau. La fille soldat, classée par l'auteur «fait historique », se situe dans la série des pièces patriotiques, quoiqu'elle ait été composée en décembre 1794, après la chute de Robespierre.

Ce revirement est d'autant plus significatif que l'auteur se consacra, juste avant cette période, aux spectacles plus légers, hérités du rire traditionnel du Théâtre-Italien, avec des 
personnages de la commedia dell'arte, comme les comédies-parades, Arlequin afficheur (avril 1792) et Colombine mannequin (février 1793), ainsi que les parodies d'œuvres sérieuses, Le projet manqué ou Arlequin taquin (mai 1792) et Arlequin Cruello (décembre 1792).

Le vaudeville s'est développé comme genre dramatique au Théâtre de la Foire aux alentours des années 1710. Le succès commercial des Forains auprès du public parisien, accru depuis l'expulsion de la Comédie-Italienne en 1697, était la cible de la jalousie du Théâtre-Français et de l'Opéra, seuls théâtres officiels ayant le privilège du roi. Les contraintes imposées par les troupes pensionnées incitèrent ce théâtre non privilégié à imaginer diverses formes dramatiques afin de déjouer leurs interdictions. Le vaudeville fut adopté dans les spectacles forains pour détourner l'interdiction de toute parole. Les vaudevilles, paroles chantées sur un air connu, se substituèrent aux années 1710 à la parole articulée par les comédiens. Cette pratique musicale permettait aux auteurs d'insister sur le sens second des paroles qui devrait être entendu par le public. L'art du vaudeville fut très en vogue dans la première moitié du dix-huitième siècle et il se remplaça par les spectacles en ariettes à partir de la seconde moitié du même siècle.

Mais le vaudeville acquit une nouvelle valeur sous la Révolution. Un article du Journal de Paris du 16 janvier 1792, en se référant à l'inauguration du Théâtre du Vaudeville, précise ainsi : « tous les autres genres de Spectacles ont eu des exemples \& des modèles chez les Peuples de l'antiquité ; le Vaudeville dut sa naissance à la gaieté française, \& il peut s'appeler, à plus juste titre que bien d'autres théâtres, Spectacle National ». On peut se rappeler également que l'article de la Gazette nationale du 11 avril 1791, que nous venons de citer, qualifie le vaudeville d'«analogue au génie de la nation ». Le vaudeville est considéré comme propriété française, s'attribuant même le statut de patrimoine national. D'ailleurs, dans un almanach de spectacle publié en 1794, l'auteur dit:

De tout temps le vaudeville a été cher aux Français ; de tout temps le vaudeville lui a servi, soit à chanter ses plaisirs, soit à peindre ses malheurs ; \&, dans l'ancien régime, il avait plus de regrets à former que de bonheur à célébrer ; aussi, comme il n'osoit pas tout dire, le vaudeville était alors aiguisé par une pointe épigrammatique, qui bien souvent, a fait pâlir le despote sous son dais, \& l'oppresseur sous sa pourpre. [...] C'est surtout depuis la Révolution que le vaudeville a repris sa force \& son véritable caractère. C'est le vaudeville qui anime nos guerriers au combat, c'est le vaudeville qui atteint sur leurs trônes les despotes ligués contre nous, \& que nos armes vont punir de leurs forfaits ; c'est enfin le vaudeville qui fait sourire nos Législateurs, au milieu des grandes affaires qu'ils traitent avec la Nature \& la Postérité. (Les spectacles de Paris, et de toute la France, 17-18) 
Il reconnaît ainsi la force et l'efficacité du vaudeville en pleine mutation sociale où les événements qui marquent l'histoire se multiplient. Ce témoignage de l'époque révolutionnaire atteste aussi du succès éminent du vaudeville et ce à peine deux ans après l'ouverture du Théâtre du Vaudeville à Paris.

\section{La pièce et le travestissement de la femme en soldat}

La pièce se compose d'un seul acte, en prose, avec des vaudevilles ou des couplets chantés. Comme nous l'avons déjà mentionné, la pièce appartient au genre des « faits historiques », très en vogue sous la Révolution et qui fait directement écho à l'actualité politique et sociale. Sa première représentation eut lieu le 13 décembre 1794, au Théâtre du Vaudeville. Cette pièce, dont l'intrigue est digne du drame bourgeois, mais avec l'enthousiasme patriotique, connut le succès avec quarante-trois représentations entre 1794 et 1795.

L'action se déroule dans une ville frontalière qui abrite le camp d'un régiment français. L'héroïne, Julie, s'est travestie en homme et a pris le nom de Victor pour s'engager comme volontaire dans la guerre contre les rois de l'Europe coalisés. Le sergent major du régiment, prénommé Julien, soupçonne que Victor est une femme, dont il est secrètement amoureux. Après avoir montré toute sa vaillance et sa valeur militaire dans le combat contre un corps d'armée autrichien qui a attaqué son régiment, le faux Victor ne peut réprimer son émotion devant la maladie qui a frappé son père inquiet de la disparition de sa fille. Son identité ainsi dévoilée malgré elle, elle décide de quitter le régiment pour retourner dans sa famille.

L'héroïne travestie en homme est un topos littéraire dont les romans et les pièces de théâtre offrent de nombreux exemples. Néanmoins, si ce type de travestissement a connu une vogue importante dans le théâtre du dix-septième siècle et de la première moitié du dix-huitième siècle, il tend à s'effacer dans la seconde moitié du dix-huitième siècle. En tant que procédé théâtral, son recul semble dû à l'émergence du drame bourgeois, qui promeut une esthétique réaliste inédite au théâtre. Mais cette éclipse prend fin aux alentours de 1780, notamment par le thème de la femme soldat, qui fait revenir la femme travestie sur la scène. La pièce de Desfontaines s'inscrit dans cet ensemble de pièces qui mettent en scène la femme déguisée en soldat. 


\section{L'effet du vaudeville dans La fille soldat}

Le vaudeville dont la particularité est le réemploi des airs connus évoque infailliblement chez le spectateur l'image du chant d'origine, et il permet d'introduire au sein de la pièce les éléments extérieurs concernant les airs employés. Ainsi Tissier dit à propos des auteurs du Théâtre du Vaudeville sous la Révolution : «Retenons seulement ici que, grâce aux couplets et au vaudeville final d'une pièce, les auteurs, quel que fût le sujet traité, ont très souvent tenu à évoquer le temps présent : tout en riant, ils surent se faire l'écho des événements » (181). Alors, quelle est la particularité de l'utilisation du vaudeville dans une pièce sous la Révolution ? Comment le contexte social de l'époque révolutionnaire a-t-il pu influencer des vaudevilles ? En prenant appui sur trois exemples d'airs utilisés dans La fille soldat, nous aborderons l'effet du vaudeville de la pièce.

Dans la pièce, on trouve trente-trois airs. Les airs employés sont très variés, de ceux employés au Théâtre de la Foire déjà aux alentours de 1710, comme «Jardinier ne vois-tu pas » ${ }^{1}$, plus connu sous le titre de l'air des « Fraises », aux chants populaires dont l'incipit figure l'amour léger, comme «Courons de la blonde à la brune », ou encore «Une fille est un oiseau », aux airs tirés des autres pièces, comme «Vaudeville de L'ile des femmes », "Vaudeville de La soirée orageuse » ou «Vaudeville de Florine ». Mais dans cet amalgame de chants, ce qui distingue la pièce des comédies en vaudevilles avant l'époque révolutionnaire, c'est l'emploi de chants patriotiques. Quel effet musical ces vaudevilles apportaient-ils à une pièce sous la Révolution ? Nous allons, pour le comprendre, tenter d'analyser les effets produits par trois vaudevilles des chants patriotiques tirés de La fille soldat: Le chant du départ, Le beau jour marqué pour la gloire et le Chant patriotique pour l'inauguration des bustes de Marat et Lepelletier. Les paroles de chaque air et vaudeville sont présentées dans le tableau à la fin de notre article.

i) Vaudeville du Chant du départ

L'air original est bien évidemment Le chant du départ, dont les paroles furent composées par Marie-Joseph Chénier et la musique par Étienne Nicolas Méhul. Écrit pour la fête du 14 juillet 1794, appelé aussi « le frère de La Marseillaise », c'est l'un des chants révolutionnaires les plus connus. Quand cette chanson a été écrite pendant l'été 1794, la France était encore dans l'engouement de la victoire à la bataille de Fleurus, remportée le 26 juin 1794. Nous apercevons la fierté confirmée par la victoire à la bataille au premier vers : «La victoire en chantant nous 
ouvre la barrière ». Après sa première exécution en public, Le chant du départ fut immédiatement imprimé à dix-huit mille exemplaires et distribué aux quatorze armées de la République ${ }^{2}$.

Il se compose de sept strophes ; la première, chantée par un représentant du peuple, est une exhortation aux soldats français qui défendent la patrie. L'appel patriotique aux soldats « La République nous appelle, / Sachons vaincre, ou sachons périr : / Un Français doit vivre pour elle ; / Pour elle, un Français doit mourir. » est repris et amplifié par le chœur des guerriers.

Dans la pièce, cet air est réemployé à la scène du départ du régiment de l'héroïne, qui se place au milieu de la pièce :

\section{JULIEN.}

J'embrasse mon ami, et je suis heureux.

AMBROISE.

I' s'fait tard, allez.

Quelques soldats arrivent à la fin de ce couplet.

\section{SCENE XI.}

Les mêmes, SOLDATS.

CHEUR.

Chant du Départ.

Le laurier nous attend, courons à la victoire ;

La Liberté veille sur nous :

[...]

La République nous appelle,

Sachons vaincre, ou sachons périr :

Un Français doit vivre pour elle ;

Pour elle, un Français doit mourir.

Un Français, etc. (29)

Dans ce vaudeville, l'auteur reprend exactement l'univers du Chant du départ, avec le même registre lexical militaire et patriotique. Pour accompagner le départ du régiment de l'héroïne, on ne pouvait en effet imaginer de chant plus approprié que Le chant du départ. La musique et les paroles font directement écho à la guerre qui appartient à la réalité des spectateurs. En reprenant délibérément le refrain de 1'air original : « La République nous appelle, / Sachons vaincre, ou sachons périr : [...] », Desfontaines incite le public à fredonner ou à chanter en même temps que les comédiens sur la scène. Comme l'a bien fait remarquer Béatrice Didier : « Le refrain permet la reprise collective du chant, tandis que les strophes, plus difficiles, seront la plupart du temps chantées par des professionnels. Le refrain a donc une valeur de propagande, il impose des slogans » (111). Telle est l'efficacité militante du vaudeville qui participe à la propagande : le 
public de théâtre est conduit à faire siens, par le plaisir d'une expérience esthétique, les slogans dictés par l'hymne officiel.

Comment le public de l'époque apprenait-il ces chants patriotiques ? En 1794, un journal se mit à paraître sous le nom de Musique à l'usage des fêtes nationales. Il reproduisait chaque mois pour ses abonnés une symphonie, un hymne ou plusieurs chansons patriotiques. Le Comité de salut public en assurait sur ses fonds la diffusion dans tous les districts, « afin [que ces airs] puissent y servir dans les fêtes civiques et de manière à les mettre à la portée du plus grand nombre des citoyens » (Pierre 24), selon un arrêté du 15 février 1794. Une maison d'édition, Magasin de musique à l'usage des fêtes nationales, a été spécialement créée pour cet objectif. La première livraison du journal date du 9 avril 1794. Le chant du départ, ainsi que les deux autres chants révolutionnaires que nous traitons ici furent imprimés et diffusés par cette maison d'édition. Le succès de ce journal amena à la création d'un autre périodique, intitulé Ouvrage périodique de chansons et romances civiques, qui présentait chaque mois quatre chansons ou romances pour une voix pour les abonnés à bas prix (Place 17).

ii) Vaudeville de l'air Le beau jour marqué pour la gloire

Dans la pièce, cette chanson accompagne le retour triomphal de Victor, qui a repris le drapeau français au soldat autrichien qui l'avait enlevé.

SCENE XIV.

VARIN, accourant. Victor, Victor...

$$
\text { Les mêmes, VARIN. }
$$

JACQUELINE, JULIEN, AMBROISE. Victor !

VARIN. Avec le drapeau... grands et petits, jeunes et vieux, tout le monde le suit.

[...]

Sur l'air suivant [=Air : Le beau jour marqué pour la gloire] Victor arrive à la tête des soldats, avec le drapeau. Julien le presse d'un côté, Jacqueline de l'autre, le village le suit. (36-37)

L'air original a pour titre «Hymne chanté par le peuple à la fête de Bara et Viala »; la musique est de Méhul, le compositeur du Chant du départ, et les paroles de Charles-Josephe Lœuillard Davrigny. Cet air est également appelé « Le beau jour marqué pour la gloire », d'après le premier vers de la chanson, ce qui nous a permis d'en retrouver la partition.

Joseph Bara et Joseph Agricole Viala, comme Lepelletier et Marat, que nous aborderons plus tard, font partie des héros de la Révolution. Ce sont tous deux des enfants engagés volontaires et morts très jeunes, Viala à l'âge de treize ans, en juillet 1793, Bara à quatorze ans, en décembre de la même année. La propagande a fait de leur mort un sacrifice pour la patrie, et a fait d'eux un 
exemple de patriotisme à l'usage des jeunes citoyens. Les honneurs du Panthéon leur furent décernés et la Convention nationale fit distribuer dans toutes les écoles primaires du pays une gravure qui représentait Bara et Viala. Viala (1780-1793), né à Avignon, était commandant de la garde nationale des jeunes avignonnais. Il a été blessé quand il voulait empêcher, à coup d'une hache, les insurgés marseillais de passer la Durance. Il a perdu sa vie à l'âge de treize ans le 6 juillet 1793. Bara (1779-1793) était en vrai un jeune domestique assassiné par des voleurs, mais la légende voulait qu'il s'engage volontairement à l'armée républicaine pour combattre les Vendéens le 7 décembre 1793, il meurt à l'âge de quatorze ans. C'est dans cette mythification de la mort de ces jeunes que l'air original a été composé pour leur fête funèbre, programmée le 28 juillet 1794. Mais la fête n'eut pas lieu suite au 9 Thermidor (27 juillet 1794), jour de la chute de Robespierre. La glorification de Bara et Viala les a placés au rang de héros immortels. Leur mort est associée à la victoire à la bataille de Fleurus (« Le peuple souverain s'avance, / Couvert des palmes de Fleurus ») que l'armée française venait de remporter le 26 juin 1794 contre la coalition. Le premier vers, «Le beau jour marqué pour la gloire », renvoie à cette date dans les annales patriotiques.

Dans la pièce de Desfontaines, l'auteur superpose Victor à l'image des jeunes martyrs de la liberté, Bara et Viala, car son exploit face à l'ennemi l'assimile à ces jeunes héros républicains. Le choix de son pseudonyme, Victor, inscrit d'ailleurs l'héroïne parmi les « fils de la victoire » de l'air original. La comparaison des derniers vers marque nettement l'influence de l'air original sur celui de la pièce de Desfontaine : «Chantons, chantons, que nos hymnes de gloire / Montent jusqu'au séjour de l'immortalité ! », à quoi répond dans l'air de Desfontaines «Victor ! Victor ! ton âme et ton courage / T'élèvent aux honneurs de l'immortalité ». Le choix de cet air permet donc à l'auteur de souligner la vaillance de Victor en rappelant l'image de Bara et Viala ; il fait sentir au public l'esprit de sacrifice qui anime l'héroïne, à l'exemple des jeunes héros de la légende.

iii) Vaudeville du Chant patriotique pour l'inauguration des bustes de Marat et Lepelletier

Cet air est d'abord inséré dans la déclaration d'amour que Julien adresse à l'héroïne à la dernière scène de la pièce. Victor s'assure de la santé de son père par la lettre et Julien profite de l'occasion pour lui avouer son amour :

VICTOR, baisant la lettre.

Oui, c'est son écriture. (A Julien.) Que mal vous m'avez fait !

JULIEN.

C'est ta faute... Achève, et tu verras que, dans tout ceci, il n'y a de vrai que sa lettre... et ma tendresse.

VICTOR. 
Votre tendresse !

JULIEN.

AIR : Du C. Gossec.

Oui mon cœur, qui dût se taire, [...]

Dans le texte de la pièce, ce vaudeville était imprimé avec la partition, mais le titre de l'air original n'était pas précisé : il était simplement mentionné «Air : Du C. Gossec » (48; voir la partition reproduite à la fin de notre article). Nous avons heureusement pu identifier l'air original. Il s'agit du « Chant patriotique pour l'inauguration des bustes de Marat et Lepelletier », dont les paroles sont d'André François de Coupigny, et la musique de François-Joseph Gossec.

Il n'est pas exagéré de dire que Marat et Lepelletier sont les deux « saints » de la Révolution française. Lepelletier (1760-1793), marquis de Saint-Frargeau, était d'abord député de la noblesse de Paris aux États généraux de 1789, mais reniant sa noblesse, il est devenu montagnard. Il faisait partie des conventionnels régicides. Son vote pour l'exécution de Louis XVI lui a valu d'être assassiné par un ancien garde du corps du roi le 20 janvier 1793. Il est alors considéré comme le «premier martyr de la Révolution » et son corps a été exposé sur la place Vendôme avant ses obsèques. Il est inhumé au Panthéon le 24 janvier.

Le culte des héros révolutionnaires, inauguré après la mort de Lepelletier, est amplifié après celle de Marat, assassiné par Charlotte Corday le 13 juillet 1793. Les funérailles de Lepelletier et de Marat sont célébrées par plusieurs sections de Paris et de la province. Des bustes à leur image sont dévoilés un peu partout. En octobre 1793, les artistes de l'Opéra se rassemblaient pour célébrer les bustes des deux martyrs dont on avait décoré la façade de l'Opéra, nommé alors «Temple des Arts et de la Liberté » (Place 190). Le chant patriotique de Gossec a été composé pour l'une de ces innombrables cérémonies. Dans cet air, Marat et Lepelletier sont présentés comme les dignes successeurs des héros de la Rome antique, Brutus (Lucius Junius Brutus), fondateur de la République romaine et un des premiers consuls romains, et les frères Gracques, réformateurs de la société romaine. Ils sont appelés «fondateurs de la Liberté » et sont érigés en modèles pour tous les citoyens de la République.

Le vaudeville de la pièce de Desfontaines a un ton tout à fait différent de la solennité de l'air original. L'air original était un hymne à la liberté, alors que celui de Desfontaines est une déclaration d'amour. La référence aux héros romains de l'air original est remplacée par une invocation à Bellone et au dieu de l'Amour, divinité féminine. L'air original chante la délivrance du peuple et de la Nation tout entière, qui ont abattu la monarchie; celui de Desfontaines ne 
concerne que les deux jeunes amoureux. La légèreté des paroles de Desfontaines fait contraste avec la gravité de l'air original qui glorifie les martyrs républicains.

La deuxième partie de l'air de notre pièce est chantée en duo par Victor et Julien sur le même air de Gossec. Ces vers de Victor « Qu'en brave, et franc militaire, Victor te donne sa foi ! Tout bas, mon sexe m'accuse, Et me dit de résister...», en regard de ceux de Julien, « Tout haut, ton habit t'excuse ", soulignent le contraste, chez l'héroïne, entre l'être et le paraître, entre sa fausse identité masculine et sa véritable identité féminine. Cette partie exprime l'hésitation de la fille travestie en homme. La décence féminine empêche Victor, alias Julie, de répondre tout de suite favorablement à la déclaration d'amour de Julien. Mais Julien incite Victor à accepter son amour en lui suggérant de s'autoriser l'audace de son habit militaire.

Les vers : (VICTOR) « Hâte-toi d'en profiter » et (JULIEN) « Hâtons-nous d'en profiter », résonnent en écho de la séduction mutuelle qui s'opère entre ces deux amoureux. La discordance entre le chant patriotique de Gossec et l'air de la pièce nous paraît aujourd'hui surprenante, comme si l'auteur voulait désacraliser l'air original en se détachant de l'idéologie républicaine. Cette discordance entre le vaudeville et l'air original est employée fréquemment au théâtre de la Foire dans la première moitié du dix-huitième siècle surtout pour des effets comiques. Mais est-ce que Desfontaines visait de la même façon à introduire un effet sarcastique à la scène finale de sa pièce patriotique ? Nous y croirons difficilement. Dans cette pièce ne se trouve aucun autre indice ironique ou parodique qui désacralise le patriotisme.

D'ailleurs, à l'époque révolutionnaire, l'utilisation des airs connus pour de nouvelles paroles qui n'avaient rien à voir avec le sens des paroles de l'air original est très fréquente. La plupart des chansons ont été créées sur un timbre connu avec lequel les citoyens ou les citoyennes se familiarisaient aisément et le fredonnaient ensuite. Ginette et Georges Marty recensent le répertoire des chansons composées sous la Révolution et conclurent ainsi : «Sur un total de 2878 chansons répertoriées entre 1789 et 1799, 188 seulement, soit 6,5\%, ont été écrites sur des musiques spécifiques. [...] Il faut bien admettre que le résultat produit par l'association d'un air contrastant, jurant même par son allure, avec le sens des paroles, n'est pas toujours heureux ; entendre chanter un Hymne en l'honneur de M. Le Peletier mort assassiné sur la mélodie de "la Marseillaise" ou La fin de Louis Capet sur "Robin ture lure” est pour le moins surprenant » (16). Les exemples sont innombrables, comme la chanson Déclaration des Droits de l'homme (1789) composée sur l'air d'amour pastoral « Philis demande son portrait ». 
La déclaration amoureuse du sergent major à la brave héroïne enrôlée volontaire chantée sur l'air républicain ne sert bien évidemment pas à la satire dans cette pièce. Il faut y voir, d'une part, un exemple typique de vaudeville détaché du contexte de l'air original et employé de manière autonome, mais aussi, d'autre part, un signe de la familiarité du public de l'époque avec les chants révolutionnaires.

L'analyse de cette pièce de Desfontaines nous a permis de mesurer l'influence que la vie sociale de l'époque révolutionnaire a pu exercer sur un type de spectacle conçu pour le divertissement du grand public. Les vaudevilles tirés des chants révolutionnaires attestent de la pénétration des musiques patriotiques dans tous les secteurs de la vie culturelle, même sur les tréteaux destinés au pur divertissement. Et si ces vaudevilles ont pu être utilisés dans un contexte très éloigné de l'air original, ce détournement même n'est qu'une preuve supplémentaire, bien que paradoxale, de la familiarité du public de l'époque avec les musiques patriotiques. 
Partition de l'air du C. Gossec de La fille soldat ${ }^{3}$

\section{Julien}

\section{AIR : du C. Gossec}
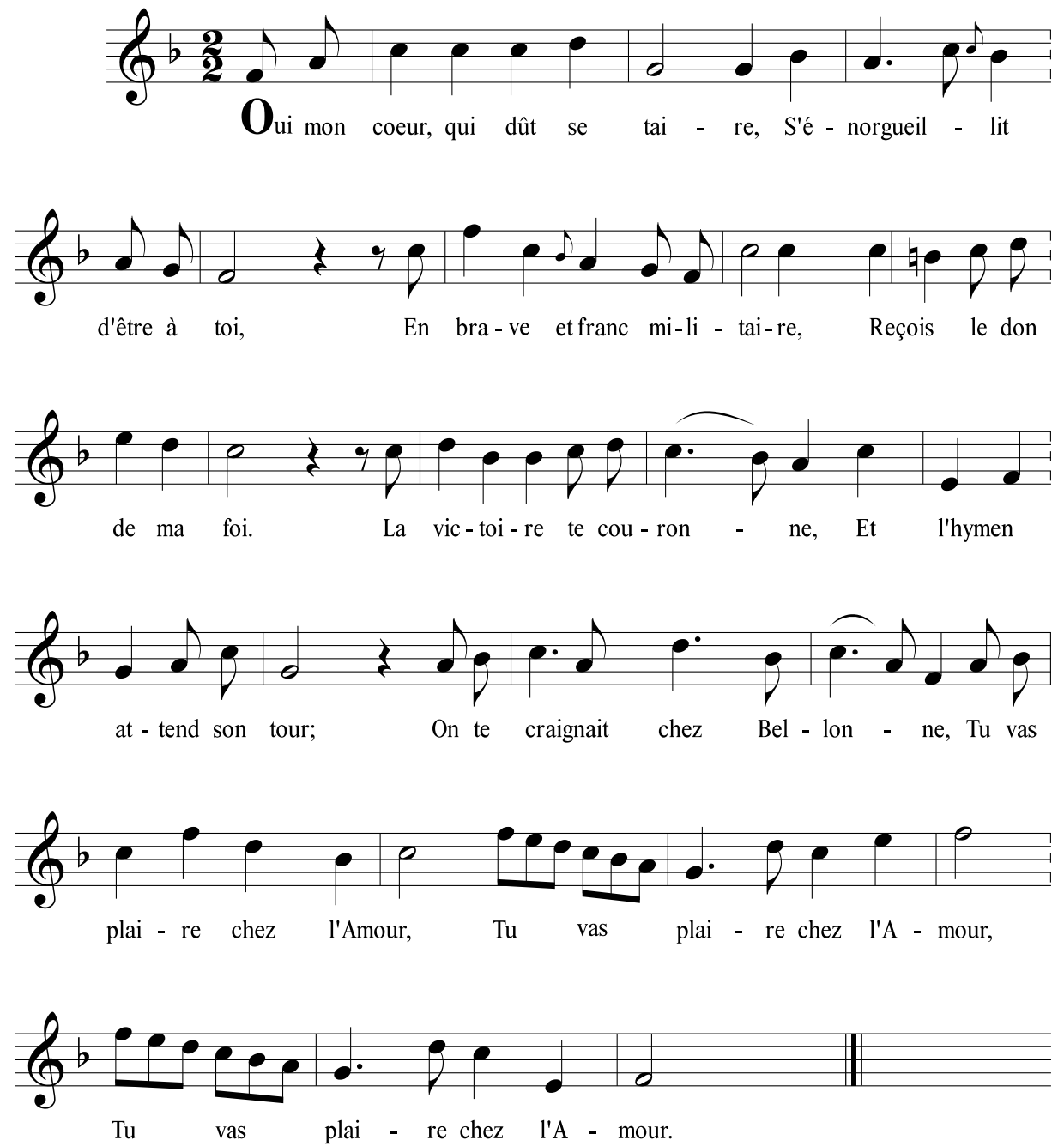
Tableau comparatif de l'air original et du vaudeville

Airs originaux

Vaudevilles dans La fille soldat

Chant du départ (Méhul)

La victoire en chantant nous ouvre la barrière

La liberté guide nos pas.

Et du Nord au Midi la trompette guerrière

A sonné l'heure des combats.

Tremblez, ennemis de la France

Rois ivres de sang et d'orgueil

Le peuple souverain s'avance

Tyrans, descendez au cercueil !

La République nous appelle,

Sachons vaincre, ou sachons périr :

Un Français doit vivre pour elle ;

Pour elle, un Français doit mourir.

Un Français, etc.
Air : Chant du départ.

Le laurier nous attend, courons à la victoire :

La Liberté veille sur nous :

Qu'à l'envi, dans nos rangs, la valeur et la gloire

Guident nos bras, portent nos coups.

Nos cœurs ont proscrit l'esclavage,

L'honneur est fixé sur nos pas,

De nos succès il est le gage ;

Marchons, affrontons le trépas.

La République nous appelle,

Sachons vaincre, ou sachons périr :

Un Français doit vivre pour elle ;

Pour elle, un Français doit mourir.

Un Français, etc.
Hymne chanté par le peuple à la Fête de

Bara et Viala (Méhul)

Le beau jour marqué par la gloire

Luit sur nos superbes remparts :

Accourez, fils de la Victoire,

Rassemblez-vous de toutes parts !

A l'auguste voix de la France,

Que les cieux répondent, émus ;

Le peuple souverain s'avance,

Couvert des palmes de Fleurus.
Air : Le beau jour marqué pour la gloire

Juste ciel ! tu viens de m'entendre,

Assure à jamais son bonheur.

A nos vœux tu viens de le rendre,

Plus de regrets, plus de douleur.

Chaqu'instant comblait nos alarmes,

Chaque mot nous ôtait l'espoir :

Après tant d'effroi, tant d'alarmes,

Ah ! qu'il est doux de te revoir ! 
Honneur à la mémoire

De Barra, de Viala, morts pour la liberté !

Chantons, chantons, que nos hymnes de gloire

Montent jusqu'au séjour de

l'immortalité !
Jouis, jouis de notre hommage :

Tu ne vis, ne combats que pour la Liberté,

Victor! Victor ! ton âme et ton courage

T'élèvent aux honneurs de l'immortalité.
Air : Du C. Gossec

JULIEN.

Oui mon cœur, qui dût se taire, S'énorgueillit d'être à toi, En brave et franc militaire, Reçois le don de ma foi.

La victoire te couronne, Et l'hymen attend son tour ;

On te craignait chez Bellonne, $\mathrm{Tu}$ vas plaire chez l'Amour, Tu vas plaire chez l'Amour, Tu vas plaire chez l'Amour,

\section{VICTOR.}

Quoi! Tu veux que sans mystère

L'amour me fasse la loi !

Qu'en brave, et franc militaire, Victor te donne sa foi !

Tout bas, mon sexe m'accuse, Et me dit de résister...

(JULIEN) Tout haut, ton habit t'excuse. 
(VICTOR) Hâte-toi d'en profiter

(ENSEMBLE) Hâtons-nous d'en profiter

(ENSEMBLE) Hâte-toi d'en profiter

\section{Bibliographie}

Carlson, Marvin. Le théâtre de la Révolution française. Tr. J. et L. Bréant. Paris : NRF-Gallimard, 1970.

Desfontaines, François-Georges. La fille soldat. Paris : Libraire au Théâtre du Vaudeville, 1794.

Didier, Béatrice. «La Révolution à la recherche d'un art total : Les Fêtes et l'Opéra ». La Révolution française et la littérature. Dir. Hisayasu Nakagawa. Kyoto : PU de Kyoto, 1992. 111.

Gazette nationale ou le Moniteur universel 101 (11 avril 1791), Réimpression de l'ancien moniteur, t. VIII. Paris : Au bureau central, 1841.

Gossec, François-Joseph. Chant patriotique pour l'inauguration des bustes de Marat et de Lepelletier, par Coupigny, des Bureaux de la Marine. Musique de Gossec. Air pour une basse taille ou tenor. Paris : Magasin de musique à l'usage des fêtes nationales, 1793.

Journal de Paris 16. 16 janvier 1792.

Marty, Ginette et Georges Marty. Dictionnaire des chansons de la Révolution. Paris : Tallandier, 1988.

Méhul, Étienne-Nicolas. Le Chant du départ, hymne de guerre. Paris : Magasin de musique à l'usage des fêtes nationales, 1794.

---. Hymne chanté par le peuple à la fête de Barra et Viala le 10 thermidor, par Davrigny..., adopté par la Commission de l'Instruction publique. Paris : Magasin de musique à l'usage des fêtes nationales, 1794.

Pierre, Constant. Le magasin de musique à l'usage des fêtes nationales et du Conservatoire. Paris : Fischbacher, 1895.

Place, Adélaïde de. La vie musicale en France au temps de la Révolution. Paris : Fayard, 1989.

Les spectacles de Paris, et de toute la France, ou Calendrier historique \& chronologique des théâtres. Paris : Duchesne, 1794.

Tissier, André. Les spectacles à Paris pendant la Révolution, répertoire analytique, chronologique et bibliographique, de la proclamation de la République à la fin de la Convention nationale (21 septembre 1792-26 octobre 1795). Paris : Droz, 2002. 


\section{NOTES}

Cet article est une version remaniée et augmentée d'une communication présentée lors du Congrès des sciences humaines du Canada en 2016. La recherche a été financée par la Société japonaise de la promotion des sciences (JSPS KAKENHI Grant Number JP24720080) que je tiens à remercier.

${ }^{1}$ Nous trouvons cet air employé dans Arlequin fille malgré lui de Pierre-François Biancolelli, pièce représentée en 1713 à la Foire Saint-Laurent. Sous le titre de l'air des « Fraises », nous remarquons plusieurs exemples. Voir « Index des airs et des vaudevilles », Théâtre de la Foire, anthologie de pièces inédites 1712-1736, dir. Françoise Rubellin, Montpellier, Espaces 34, 2005, 410.

${ }^{2} \mathrm{http}: / /$ www.chansons-net.com/histoire/index.php?param1=MI0111.php

${ }^{3}$ Je sais gré à Élise Droneaud d'avoir retranscrit pour cet article la partition de l'air du C. Gossec de La Fille soldat (48-49). 